\title{
Mean Bit Error Rate Analysis of High Rate IEEE 802.15.3.a UWB Channels
}

\author{
J. S. Daba \\ University of Balamand, Koura, Lebanon
}

Received October 20, 2020; Revised December 18, 2020; Accepted December 30, 2020

\begin{abstract}
Cite This Paper in the following Citation Styles
(a): [1] J. S. Daba, "Mean Bit Error Rate Analysis of High Rate IEEE 802.15.3.a UWB Channels," Universal Journal of Electrical and Electronic Engineering, Vol. 7, No. 6, pp. 328 - 338, 2020. DOI: 10.13189/ujeee.2020.070605.
\end{abstract}

(b): J. S. Daba (2020). Mean Bit Error Rate Analysis of High Rate IEEE 802.15.3.a UWB Channels. Universal Journal of Electrical and Electronic Engineering, 7(6), 328 - 338. DOI: 10.13189/ujeee.2020.070605.

Copyright $\bigcirc 2020$ by authors, all rights reserved. Authors agree that this article remains permanently open access under the terms of the Creative Commons Attribution License 4.0 International License

\begin{abstract}
In this work, the performance of ultra-wide band systems (UWB) in high-speed wireless networks is studied. At the physical layer of wireless personal area networks, dual carrier modulation driving multiband orthogonal frequency division multiplexing is implemented for $480 \mathrm{Mbps}$ data rates over 4 classes of UWB scattering channels (CM1, CM2, CM3, CM4) bundeled as per the IEEE 802.15.3a UWB standard. Generalized wireless fading models are presented and canonical expressions for mean bit error rates (MBER) are derived for different modulation and receiver diversity combining schemes. A generating model in terms of the characteristic function of the signal-to-noise ratio is introduced for MBER under general statistical fading conditions, which further develops our previous work. The power density spectral characteristic of the multi-user noise is tuned to a novel generalized innovation-matched filter (GIMF) which is at the core of the UWB receiver. Because of its robustness at also capturing the fading characteristics of the UWB channels, the GIMF detector yields better performance than the classical matched filter and a 10-finger RAKE receiver. The relatively flat CM1 channel is proven to have the best performance, while the highly frequency selective CM4 suffers from the worst performance. CM3 channel slightly outperforms CM4 channel between 0 and $5 \mathrm{~dB}$ and exhibits significant improvement over CM4 above $5 \mathrm{~dB}$ SNR. CM3 and CM4 channels are proven to have nearly identical performance below $0 \mathrm{~dB}$ margins. A comparative analysis was also conducted for the MBER of our model and that of the CF-based model at a 10-finger RAKE receiver developed by Wang et al. It was found that our model outperforms
\end{abstract}

that of the CF-model for CM1 and CM2 channels for a wide dynamic range of SNR values. For CM3 and CM4 channels, our model's performance was superior for SNR values below $8 \mathrm{~dB}$.

Keywords Bit Error Rate, Generalized Innovation-Matched Filter, RAKE Receiver, Ultra-Wide Band

\section{Introduction}

Ultra-wide band initially gained popularity in wireless personal areal networks for short range applications [1-7]. With the recent proliferation of $6 \mathrm{G}$ networks research, UWB systems are proposed for usage at sub-6G spectral bands with applications to wireless body area networks and the Internet of Things.

As a fundamental rule, the bandwidth $(B W)$ of a UWB signal with center frequency $f_{c}$ obeys the constraint

$$
B W>25 \% f_{c} \text {. }
$$

In a broad sense, two types of UWB systems are deployed, Direct Sequence Ultra-Wide Band and Multi-Band Orthogonal Frequency Modulation, with the latter enjoying superior performance and wider usage [8-14]. The IEEE 802.15.3.a UWB standard describes 4 classes of channels named CM1, CM2, CM3 and CM4. The characteristics of the said channels are describes in Table 1 in terms of the scattering fading statistics and the transmission ranges. 
Table 1. IEEE 802.15.3a UWB channels

\begin{tabular}{|c|c|c|c|c|}
\hline & CM1 & CM2 & CM3 & CM4 \\
\cline { 2 - 5 } & $\begin{array}{c}\text { Line of } \\
\text { sight } \\
\text { (LOS) }\end{array}$ & $\begin{array}{c}\text { Non-line } \\
\text { of Sight } \\
\text { (NLOS) }\end{array}$ & NLOS & NLOS \\
\hline $\begin{array}{c}\text { Common } \\
\text { Fading } \\
\text { Statistics }\end{array}$ & Rician & Rayleigh & Nakagami-m & Lognormal \\
\hline $\begin{array}{c}\text { Transmission } \\
\text { Range }\end{array}$ & $\begin{array}{c}0 \text { to } \\
4 \mathrm{~m}\end{array}$ & 0 to $4 \mathrm{~m}$ & 4 to $10 \mathrm{~m}$ & 4 to $10 \mathrm{~m}$ \\
\hline
\end{tabular}

\section{Stochastic Modeling of UWB Channels}

For better accuracy, we consider the more realistic partially developed scattering model [15-25] where the received signal is modeled in passband form according to a random walk in a two-dimensional plane [26-36]

$$
r(t)=\left|\sum_{k=1}^{N} A_{k} e^{j \phi_{k}}\right| s(t)+n(t),
$$

where $\left(A_{k}, \phi_{k}\right)$ are the amplitude and phase of the $k$-th blocking scattering element, respectively, $N$ is the number of scatterering elements in the channel, $s(t)$ is the modulated signal, and $n(t)$ is the additive white and multi-user colour noise. This stochastic model conforms to $\mathrm{CM} 2, \mathrm{CM} 3$, and $\mathrm{CM} 4$ channels.

When a direct LOS specular component with power | $V_{0}^{2} \mid$ is added to the diffuse component, the received signal model takes the generalized form

$$
r(t)=\left|\sum_{k=1}^{N} A_{k} e^{j \phi_{k}}+V_{0}\right| s(t)+n(t), N \gg .
$$

CM1 channels obey this law where the statistical distribution of the instantaneous signal-to-noise ratio (SNR) is a modified-Rician distribution. In the absence of a direct LOS specular component, Nakagami- $m$ statistics enjoy widespread fading applications where the instantaneous SNR is gamma-distributed with shaping parameter $m$.

A more severe fading model follows the exponential law

$$
r(t)=\exp \left(\sum_{k=1}^{N} A_{k}\right) s(t)+n(t), N \gg,
$$

in which case the instantaneous SNR is lognormally distributed. This large scale shadowed scattering is well suited for CM3 and CM4 channels.

Yet another more realistic fading model is termed partially developed mobile fading. In this stochastic model, the number of scatterers $N$ is very small and the received signal in this environment is modeled in complex baseband form as

$$
\tilde{r}(t)=\left|\sum_{k=1}^{N} A_{k} e^{j \phi_{k}}\right| e^{j 2 \pi \xi_{D}} \tilde{s}(t)+n(t),
$$

where $\xi_{D}$ is the Doppler shift which is randomly distributed over the support $\left[-F_{D}, F_{D}\right]$, the maximum Doppler shift $F_{D}$ is given by the well know wave propagation formula

$$
F_{D}=\left|v_{R}\right| f_{c} /(\delta c), \delta \leq 1,
$$

$c$ being the speed of light, $v_{R}$ the relative velocity between transmitter and receiver, and $\delta$ the wave attenuation constant which depends on the channel scattering characteristics. CM3 and CM4 channels are well-suited for this model.

\section{System Performance Metrics}

\subsection{Generalized Innovation-Matched Detector}

The receiver structure comprises a generalized innovation-line match filter (GIMF) to capture the statistical characterisitcs of the UWB multipath scattering channels and to spectrally shape the additive colour noise (ACN) caused by multi-user interference. The realizable transfer function of the GIMF, which can be considered as a pre-equalizer to the classical UWB RAKE receiver [37], is given by

$$
H_{o}(f)=P_{S}^{-}(f) e^{-j 2 \pi f / R_{b}} / P_{N}(f),
$$

where $P_{N}(f)$ is the power spectral density (PSD) of the ACN, $R_{b}$ is the coding rate, and

$$
P_{S}(f)=P_{S}^{+}(f) P_{S}^{-}(f),
$$

is the separable PSD of the modulation signal, which is the synthesis of the mixture of its causal and non-causal components $P_{S}^{+}(f)$ and $P_{S}^{-}(f)$, respectively, where $P_{S}^{-}(f)=P_{S}^{+^{*}}(f)$. If the PSD separability condition fails, we set $P_{S}^{-}(f)=\sqrt{P_{S}(f)}$, resulting in the sub-optimal transfer function

$$
H_{o}(f)=\sqrt{P_{S}(f)} e^{-j 2 \pi f / R_{b}} / P_{N}(f) .
$$

The term "innovation" is used for the GIMF structure because

$$
\left|H_{o}(f)\right|=\frac{2}{N_{0}}\left|P_{S}^{+}(f)\right|
$$

for white noise with PSD $N_{0} / 2 \quad$ [Watts $/ \mathrm{Hz}$ ), and $P_{S}^{+}(f)$ acts as an innovation filter for the modulation signal $s(t)$.

The resulting optimal signal-to-noise ratio of the received filtered signal is given by 


$$
S N R_{0, \max }=\int_{-\infty}^{\infty} P_{S}(f) / P_{N}(f) d f .
$$

Further to the PSD separability condition of Eq. (8), it can be proven that a signal $\mathrm{x}(\mathrm{t})$ with square-integrable spectral density is regular if and only if its PSD $P_{X}(f)$ obeys the Paley-Wiener (PW) condition

$$
\int_{-\infty}^{\infty}\left|\log \left(P_{X}(w)\right)\right| /\left(1+w^{2}\right) d w<\infty .
$$

Thus, the power spectral density of a regular process $x(t)$ is always separable as the mixture of its non-causal and causal components.

\subsection{Energy Detection Performance}

In digital communication systems, the signal-to-noise ratio

$$
\Gamma_{0} \triangleq E_{b} / N_{0},
$$

is considered as a reference performance metric, where $E_{b}$ is the energy of the modulation signal per bit and $N_{0}$ is the PSD of the additive white noise (AWGN). All performance measures, such as the MBER, the outage probability, the average outage duration etc ... are functions of the SNR $\Gamma_{0}$.

Since UWB signals are subject to multipath fading noise, a more appropriate metric is the average of the instantenous SNR per bit

$$
\bar{\Gamma}=E\left(\gamma^{2}\right) E_{b} / N_{0},
$$

where $\gamma$ is the fading noise envelope, $\gamma^{2}$ is the fading noise power, and $P_{\text {dif }}=E\left(\gamma^{2}\right) \leq 1$ is the mean diffuse power.

To study the performance of energy detectors, it is necessary to obtain the analytical expression of the mean probability of detection, or equivalently the MBER, as per the equation

$$
\overline{P_{e}}=E_{\Gamma}\left[P_{e}(\Gamma)\right]=\int_{0}^{\infty} P_{e}(v) p_{\Gamma}(v) d v,
$$

where $P_{e}(\Gamma)$ is the BER in an AWGN channel free of multipath fading and $p_{\Gamma}(v)$ is the probability distribution of $\Gamma$.

Evaluation of Eq. (15) for a particular form of the bit error probability comprising a Gaussian-Q function (in AWGN), requires knowledge of the definite integral

$$
I=\int_{0}^{\infty} Q(\alpha \sqrt{v}) p_{\Gamma}(v) d v .
$$

After considerable manipulations, Eq. (16) can be rewritten as

$$
I=\frac{1}{\pi} \int_{0}^{\pi / 2} M_{\Gamma}\left(-\alpha^{2} /\left(2 \sin ^{2} t\right)\right) d t,
$$

where $M_{\Gamma}($.$) is the moment generating function of the$ random variable $\Gamma$, defined by

$$
M_{\Gamma}(s) \triangleq E_{\Gamma}[\exp (j s \Gamma)]=\int_{0}^{\infty} e^{j v s} p_{\Gamma}(v) d v
$$

Table 2 depicts analytical expressions for the BER as a function of $\Gamma_{0}$ and the MBER in terms of $\bar{\Gamma}$ for different modulation methods. The following special functions are used: $Q($.$) is the Gaussian-Q function,$ $Q_{L}(\alpha, \beta)$ is the Marcum-Q function, $\Gamma($.$) is the$ Gamma function, and $I_{0}($.$) is the modified Bessel$ function of the $1^{\text {st }}$ kind. 
Table 2. BER and MBER analytical expressions for different modulation schemes in Rayleigh fading channels

\begin{tabular}{|c|c|c|}
\hline Modulation & Applications & MBER (Rayleigh fading) \\
\hline $\begin{array}{l}\text { Coherent } \\
\text { BPSK }\end{array}$ & $\begin{array}{l}\text { Direct } \\
\text { Sequence } \\
\text { UWB } \\
\end{array}$ & $0.5(1-\sqrt{\bar{\Gamma} /(\bar{\Gamma}+1)}) \approx 1 / 4 \bar{\Gamma}$ for $\bar{\Gamma} \gg$ \\
\hline $\begin{array}{l}\text { Coherent } \\
M \text {-PSK }\end{array}$ & $\begin{array}{l}\text { Generaliation } \\
\text { of BPSK }\end{array}$ & $\frac{1}{M}\left(1-\sqrt{M \bar{\Gamma} \sin ^{2}(\pi / M) /\left(M \bar{\Gamma} \sin ^{2}(\pi / M)+2\right)}\right)$ \\
\hline $\begin{array}{c}\text { Non-coherent } \\
\text { DPSK }\end{array}$ & $\begin{array}{l}\text { Multi-Band } \\
\text { UWB }\end{array}$ & $0.5(1+\bar{\Gamma})^{-1}$ \\
\hline \multirow[b]{2}{*}{$\begin{array}{l}\text { Non-coherent } \\
\text { DQPSK } \\
(M=4)\end{array}$} & $\begin{array}{l}\text { Multi-Band } \\
\text { UWB }\end{array}$ & $\overline{F_{\bar{\Gamma}}}(5 \pi / 4)-\overline{F_{\bar{\Gamma}}}(\pi / 4)[38],[39]$ \\
\hline & & $\overline{F_{v}}(\psi \mid M) \triangleq-\frac{\sin \psi}{4 \pi} \int_{-\pi / 2}^{\pi / 2}(1-\cos \psi \cos \xi)\left(1+v\left(\log _{2} M\right)(1-\cos \psi \cos \xi)\right)^{-1} d \xi$ \\
\hline $\begin{array}{c}\text { Non-coherent } \\
M \text {-DPSK } \\
\end{array}$ & \multirow{4}{*}{$\begin{array}{l}\text { Multi-Band } \\
\text { UWB } \\
\text { Generalized } \\
\text { DPSK and } \\
\text { DQPSK }\end{array}$} & $\frac{2}{2}\left(\overline{F_{\bar{\Gamma}}}(13 \pi / 8)-\overline{F_{\bar{\Gamma}}}(\pi / 8)\right)$ \\
\hline$M=8$ & & \\
\hline$M=16$ & & $0.25\left(\overline{F_{\bar{\Gamma}}}(15 \pi / 16)+\overline{F_{\bar{\Gamma}}}(13 \pi / 16)-\overline{F_{\bar{\Gamma}}}(11 \pi / 16)-\overline{F_{\bar{\Gamma}}}(9 \pi / 16)\right)-0.5\left(\overline{F_{\bar{\Gamma}}}(3 \pi / 16)+\overline{F_{\bar{\Gamma}}}(\pi / 16)\right)$ \\
\hline$M=32$ & & $\begin{array}{l}0.1\left(3 \overline{F_{\bar{\Gamma}}}(31 \pi / 32)+3 \overline{F_{\bar{\Gamma}}}(29 \pi / 32)-\overline{F_{\bar{\Gamma}}}(27 \pi / 32)-\overline{F_{\bar{\Gamma}}}(25 \pi / 32)+\overline{F_{\bar{\Gamma}}}(23 \pi / 32)+\overline{F_{\bar{\Gamma}}}(21 \pi / 32)\right. \\
\left.-3 \overline{F_{\bar{\Gamma}}}(19 \pi / 32)-3 \overline{F_{\bar{\Gamma}}}(17 \pi / 32)\right)+0.2\left(\overline{F_{\bar{\Gamma}}}(15 \pi / 32)+\overline{F_{\bar{\Gamma}}}(13 \pi / 32)-\overline{F_{\bar{\Gamma}}}(11 \pi / 32)-\overline{F_{\bar{\Gamma}}}(9 \pi / 32)\right) \\
-0.4\left(\overline{F_{\bar{\Gamma}}}(3 \pi / 32)+\overline{F_{\bar{\Gamma}}}(\pi / 32)\right)\end{array}$ \\
\hline $\begin{array}{c}\text { BPSK-driven } \\
\text { Direct } \\
\text { Sequence } \\
\text { CMDA } \\
\text { (DS-CDMA) } \\
\text { [40] } \\
\text { Multicode } \\
\text { (MCD) } \\
\text { scheme [41] }\end{array}$ & & $\begin{array}{l}2^{-(N K+1)} \sum_{p=-N K}^{N K} \frac{(N K) !}{\Gamma(1+0.5(N K-p)) \Gamma(1+0.5(N K+p))} \times\left(1-(K-|p / N|) \sqrt{\bar{\Gamma} /\left(1+(K-|p / N|)^{2} \bar{\Gamma}\right)}\right. \\
\approx Q\left(\Psi_{N}(\bar{\Gamma})^{-1 / 2}\right)+\left(8 \sqrt{2 \pi} \bar{\Gamma}^{-5} \sqrt{\Psi_{N}^{3}(\bar{\Gamma})}\right)^{-1} \times\left(2-\left(3+\Psi_{N}^{-1}(\bar{\Gamma})\right) /\left(4 \bar{\Gamma} \Psi_{N}(\bar{\Gamma})\right)\right), \\
\Psi_{N}(\bar{\Gamma})=(K-1) /(3 N)+0.5 / \bar{\Gamma}, N=\text { nb. of interferers; } K=\text { processing gain }\end{array}$ \\
\hline
\end{tabular}

BPSK-Driven Root-Mean-Square-Gain Combined Signals. We now consider BPSK over independent Rayleigh fading (slow and non frequency selective) diversity channels with AWGN. In a single-input-multiple-output (SIMO) channel, the SNRs of asymptotic root-mean-square-gain-combining (RMSGC) [42], [43] and maximal ratio combining (MRC) obey the same statistics as the fading power. In other words, $\Gamma$ follows a Gamma distribution with shaping parameter $L$ and scaling parameter $\bar{\Gamma}$. Considering the case where the BER is evaluated at the average operating condition, Jensen's inequality yields an MBER ( $\overline{\mathrm{BER}})$ upper bound

$$
\sup \left(\overline{\mathrm{BER}}_{\mathrm{RMSGC}}\right)=0.5(1-\sqrt{L \bar{\Gamma} /(1+L \bar{\Gamma})})
$$

We now consider the exact error probability in which the BER is averaged over all possible operating conditions by keeping these operating conditions random in order to capture their statistical fluctuations. Rigorous mathematical analysis yields the expression

$$
\overline{\mathrm{BER}}_{\mathrm{RMSGC}}=\frac{1}{2(L-1) !}\left([L-1]_{L-1}-\sqrt{\frac{\bar{\Gamma}}{\pi}}(1+\bar{\Gamma})^{\frac{1}{2}-L} \sum_{l=0}^{L-1}[L-1]_{l} \Gamma\left(L-l-\frac{1}{2}\right)(1+\bar{\Gamma})^{l}\right)=\frac{1}{2}\left(1-\left(1+\frac{L}{\bar{\Gamma}}\right)^{-\frac{1}{2}} \sum_{l=0}^{L-1}\left(\begin{array}{c}
2 l \\
l
\end{array}\right)\left[4\left(1+\frac{\bar{\Gamma}}{L}\right)\right]^{-l}\right)
$$

for $\bar{\Gamma} \geq \bar{\Gamma}_{t h}$, where $[N]_{M}$ is the reverse-Pochhammer symbol and $\Gamma($.$) is the gamma function (not to be confused with$ the reference SNR “ $\Gamma$ ”). 
For MRC, the exact expression of MBER for independent Nakagami- $m$ faded diversity signals can be expressed as:

$$
\overline{\mathrm{BER}}_{\mathrm{MRC}}=(1-\Omega)^{m L} \sum_{l=0}^{m L-1}\left(\begin{array}{c}
m L-1+l \\
l
\end{array}\right) \Omega^{l},
$$

where $\Omega=0.5(1+\sqrt{\Gamma /(1+\bar{\Gamma})})$. For Rayleigh fading, we simply set $m=1$.

BPSK-Driven Equal Gain Combined Signals. Using the Jensen's inequality, the resulting MBER upper limit for EGC is

$$
\begin{gathered}
\sup \left(\overline{\mathrm{BER}}_{\mathrm{EGC}}\right)= \\
=\frac{1}{2}(1-\sqrt{L \bar{\Gamma}(1+(L-1) \pi / 4) /(1+L \bar{\Gamma}(1+(L-1) \pi / 4))})
\end{gathered}
$$

A simple expression for the theoretical bit error rate for EGC with BPSK modulation is shown below:

$$
\begin{gathered}
\overline{\mathrm{BER}}_{\mathrm{FGC}}=\frac{1}{2}-\frac{1}{\pi} \sum_{m=1}^{M} \omega_{m} G\left(z_{m}\right), \\
G(z)=\operatorname{Im}\left\{\left[{ }_{1} F_{1}(-0.5,0.5 ; \bar{\Gamma} /(L(1+\bar{\Gamma})))+\right.\right. \\
\left.+j z \sqrt{\pi \bar{\Gamma} /(L(1+\bar{\Gamma}))}]^{L}\right\} z^{-1},
\end{gathered}
$$

$z_{m}$ are the positive zeros of the Hermite polynomial of order $M$ (typically $M=15$ is sufficient), $w_{m}$ are their corresponding weights and ${ }_{1} F_{1}($.$) is the Kummer$ confluent hypergeometric distribution. However, we have been unable to independently verify the accuracy of this expression for mean SNR values $\bar{\Gamma}$ above $15 \mathrm{~dB}$ using Monte Carlo simulations of the MBER.

A more complicated theoretical MBER expression for independent Nakagami- $m$ distributed fading branches can be also expressed in the form of a single finite-range integral and an integrand composed of tabulated transcendental functions involving Hermite polynomials. The procedure to determine $\overline{\mathrm{BER}}_{\mathrm{EGC}}=\bar{P}_{e} \quad$ is summarized below:

$$
\begin{gathered}
\overline{P_{e}}=\left(2 \pi^{2}\right)^{-1} \int_{0}^{\pi / 2} \eta(\phi)^{-1 / 2} \sum_{n=1}^{N} H_{x_{n}} \Psi\left(x_{n} / \sqrt{\eta(\phi)}, \varphi\right) d \phi, \\
\Psi(\nu, \varphi)=R(v, \varphi) \cos (\Theta(v, \varphi)), \\
R(v, \phi)=\sqrt{X^{2}(\phi)+Y^{2}(\phi)} \prod_{l=1}^{L} \sqrt{A_{l}^{2}(v)+B_{l}^{2}(v)}, \\
X(\phi)=\sqrt{\frac{2}{\pi}} \frac{\sin \phi}{D}, D=\sqrt{2 E_{b} / \sum_{l=1}^{L} N_{l}}
\end{gathered}
$$

$$
\begin{gathered}
Y(v, \phi)=-v(\sin \phi / D)^{2}{ }_{1} F_{1}\left(0.5 ; 1.5 ; 0.5(v \sin \phi / D)^{2}\right),(29) \\
A_{l}(v)={ }_{1} F_{1}\left(0.5-m_{l} ; 0.5 ; v^{2} P /\left(4 m_{l}\right)\right), \\
B_{l}(v)= \\
=\Gamma\left(m_{l}+0.5\right) / \Gamma\left(m_{l}\right) \sqrt{P_{l} / m_{l}} v_{1} F_{1}\left(1-m_{l} ; 1.5 ; v^{2} P_{l} /\left(4 m_{l}\right)\right), \quad(31) \\
\Theta(v, \phi)=\tan ^{-1}(Y(v, \phi) / X(\phi))+\sum_{l=1}^{L} \tan ^{-1}\left(B_{l}(v) / A_{l}(v)\right)+ \\
+\frac{\pi}{2}\left(L+1-\operatorname{sgn}(Y(\nu, \phi))-\sum_{l=1}^{L} \operatorname{sgn}\left(B_{l}(v)\right)\right), \\
\eta(\phi)=0.5 \sin ^{2} \phi / D^{2}+0.25 \sum_{l=1}^{L} P_{l} / m_{l},
\end{gathered}
$$

$P_{l}=E\left(\gamma_{l}^{2}\right)$ is the mean received fading power on the $l$-th branch, $E_{b}=A_{c}^{2} / 2 R_{b}$ is the energy per bit of the transmitted signal, $N_{l} / 2$ is the power spectral density of the AWGN on $l$-th branch, $m_{l}$ is the fading severity parameter of the $l$-th branch, $x_{n}$ are the zeros of the $N_{p}$-order Hermite polynomial $H_{N_{p}}(x)$, and $H_{x_{n}}$ are its weight factors given by

$$
H_{x_{n}}=2^{N_{p}-1} N_{p} ! \sqrt{\pi} /\left(N_{p}^{2} H_{N_{p}-1}^{2}\left(x_{n}\right)\right) .
$$

For Rayleigh fading with uniform diffuse fading power profile and uniform noise power spectral density profile across the diversity branches, we simply set $m_{l}=1, N_{l}=$ $N_{0}$, and $P_{l}=P_{\text {dif }}$.

Since the above form for the EGC MBER is computationally intensive (due to the presence of a Kummer confluent hypergeometric function inside the integration), we approximate its mean BER using the Delta method:

$$
\begin{gathered}
\overline{\mathrm{BER}}_{\mathrm{EGC}}=E\left(Q\left(\sqrt{2 \Gamma_{\mathrm{EGC}}}\right)\right) \approx Q\left(\sqrt{2 E\left(\Gamma_{\mathrm{EGC}}\right)}\right)+ \\
\left.0.5 \sigma_{\Gamma_{\mathrm{EGC}}^{2}}^{2} Q^{\prime \prime}(\sqrt{2 x})\right|_{x=E\left(\Gamma_{\mathrm{EGC}}\right)^{\prime}} \\
E\left(\Gamma_{E G C}\right)=E_{b} M_{\beta_{B G C}}^{\prime}(0) / N_{0}, \\
\sigma_{\Gamma_{E G C}}^{2}=E_{b}^{2} M_{\beta_{E G C}}^{(4)}(0) / N_{0}^{2}-E^{2}\left(\Gamma_{E G C}\right)
\end{gathered}
$$

where $M_{\beta_{\mathrm{EGC}}}(s)$ is the moment generating function of the EGC fading power and

$$
Q^{\prime \prime}(\sqrt{2 x})=0.5 \exp (-x)\left(1+x^{-1}\right) / \sqrt{\pi x} .
$$

Eq. (35) is obtained by a one-step Taylor approximation of $Q\left(\sqrt{2 \Gamma_{\mathrm{EGC}}}\right)$ about the mean $E\left(\Gamma_{\mathrm{EGC}}\right)$. The approximation is good if $E\left(\Gamma_{E G C}\right)$ has a high probability of being close to its mean (i.e. Amount of Fading (AoF) is small). 
Dual Carrier Modulation. A separate study is devoted for DCM since it is the employed Multi-Band UWB modulation in this research. DCM's superiority over QPSK is widely accepted in the literature [44], [45] for high rate UWB systems. We establish tight upper bounds on the BER since a closed form canonical BER expression cannot be obtained for DCM-detection. Assuming a Rayleigh fading environment, the MBER expression is upper bounded by

$$
\begin{aligned}
& \overline{P_{b}}(E) \leq 0.25\left[( \overline { \Gamma _ { 2 } } - 4 \overline { \Gamma _ { 1 } } ) ^ { - 1 } \left(4 \overline{\Gamma_{1}} \sqrt{4 \overline{\Gamma_{1}} /\left(4 \overline{\Gamma_{1}}+5\right)} \div:\right.\right. \\
& \left.+\overline{\Gamma_{2}} \sqrt{\overline{\Gamma_{2}} /\left(\overline{\Gamma_{2}}+5\right)}\right)+\left(9 \overline{\Gamma_{2}}-\overline{\Gamma_{1}}\right)^{-1}\left(\overline{\Gamma_{1}} \sqrt{\overline{\Gamma_{1}} /\left(\overline{\Gamma_{1}}+5\right)}-\right. \\
& \left.-9 \overline{\Gamma_{2}} \sqrt{9 \overline{\Gamma_{2}} /\left(9 \overline{\Gamma_{2}}+5\right)}\right)+\left(4 \overline{\Gamma_{2}}-\overline{\Gamma_{1}}\right)^{-1}\left(\overline{\Gamma_{1}} \sqrt{\Gamma_{1} /\left(\overline{\Gamma_{1}}+5\right)}-\right. \\
& \left.\left.-4 \overline{\Gamma_{2}} \sqrt{4 \overline{\Gamma_{2}} /\left(4 \overline{\Gamma_{2}}+5\right)}\right)-3\right], \overline{\Gamma_{l}}=\overline{\gamma_{l}^{2}} E_{b}^{(l)} / N_{0}, l=1,2 .
\end{aligned}
$$

For two symmetric channels, $\overline{\Gamma_{1}}=\overline{\Gamma_{2}}=\bar{\Gamma}$, Eq. (38) simplifies into

$$
\begin{gathered}
\bar{P}_{b}(E) \leq \frac{1}{4}\left(3+\frac{19}{24} \sqrt{\Gamma /(\bar{\Gamma}+5)}-\right. \\
\left.-\frac{8}{3} \sqrt{4 \bar{\Gamma} /(4 \bar{\Gamma}+5)}-\frac{9}{8} \sqrt{9 \bar{\Gamma} /(9 \bar{\Gamma}+5)}\right) .
\end{gathered}
$$

\section{Simulation and Results}

\subsection{MBER of the GIMF Receiver}

The performance measure MBER is undoutebly the hardest metric to calculate, yet, it is the most revealing criteria about the system's behavior and the one most often deployed in performance evaluation studies. For simulation purposes, MC-OFDM with DCM modulation is implemented. The receiver structure comprises the GIMF detector described in sec. 3.1 and the received UWB signal conforms to the IEEE 802.15.3a channels standard presented in the introduction section and in section 2 .

The received signal at the input of the GIMF and the filtered signal at the output of the GIMF are depicted in Fig 1 for 480 Mbps-DCM in a Rayleigh fading CM4 channel and ACN with a Gaussian PSD. It is observed that GIMF exhibits a smoothing effect on the scattered multipath signal, and consequently, the amount of fading (mean-to-standard deviation ratio of the instantaneous SNR [46-48]) is increased, thus causing a reduction in the MBER and an enhancement in the average system throughput.

The synthetic and theoretical MBER graphs are displayed in Fig 2 in terms of the average SNR per bit. The small discrepancy between empirical and theoretical MBER curves for CM4 channel is caused by the high standard deviation of the CM4 fading power.

Assuming the same noise conditions, the 480 Mbps-DCM signal is detected using the classical matched filter (CMF) in order to compare the resulting MBER values to the ones obtained using our novel GIMF structure. The empirical MBER curves are shown in Fig 3. It is evident from Fig 3 that the GIMF performance is superior to that of the CMF (all controlling parameters kept the same). This is attributed to the innovation filter characteristics of the GIMF which adaptively shapes the PSD of the ACN.

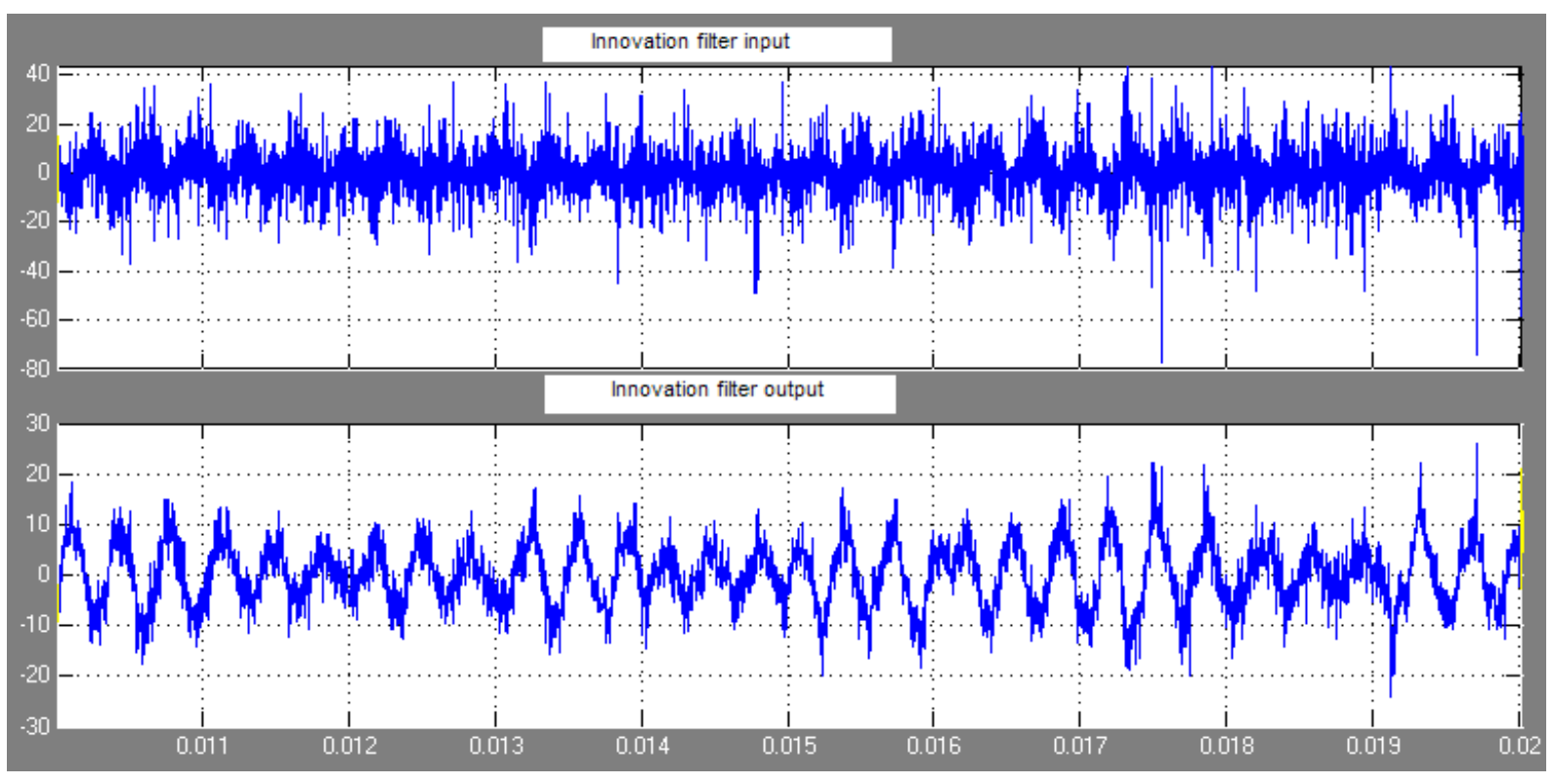

Figure 1. Received and processed UWB signals at the input and output of GIMF receiver with ACN in Rayleigh faded CM4 


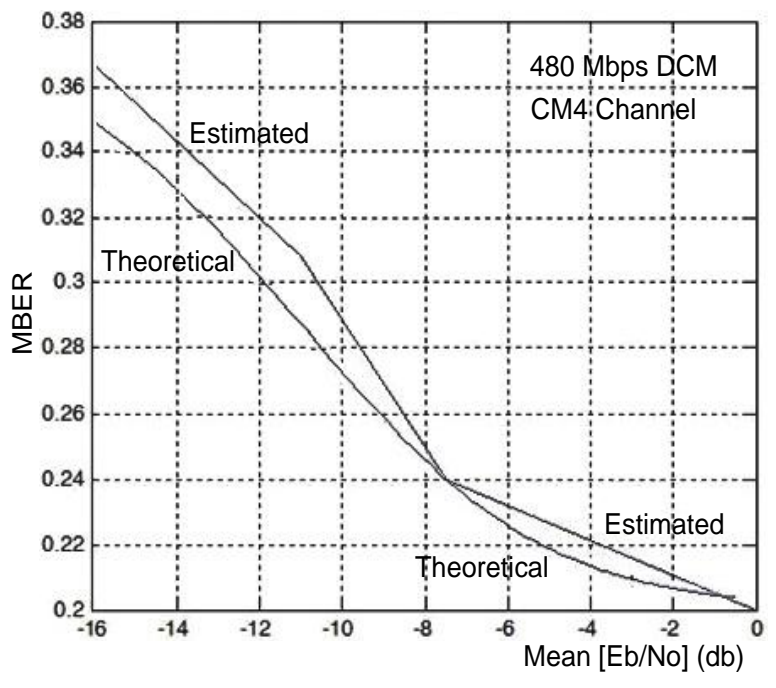

Figure 2. Empirical vs theoretical MBER for the GIMF in a Rayleigh faded CM4 channel with ACN and Gaussian PSD.

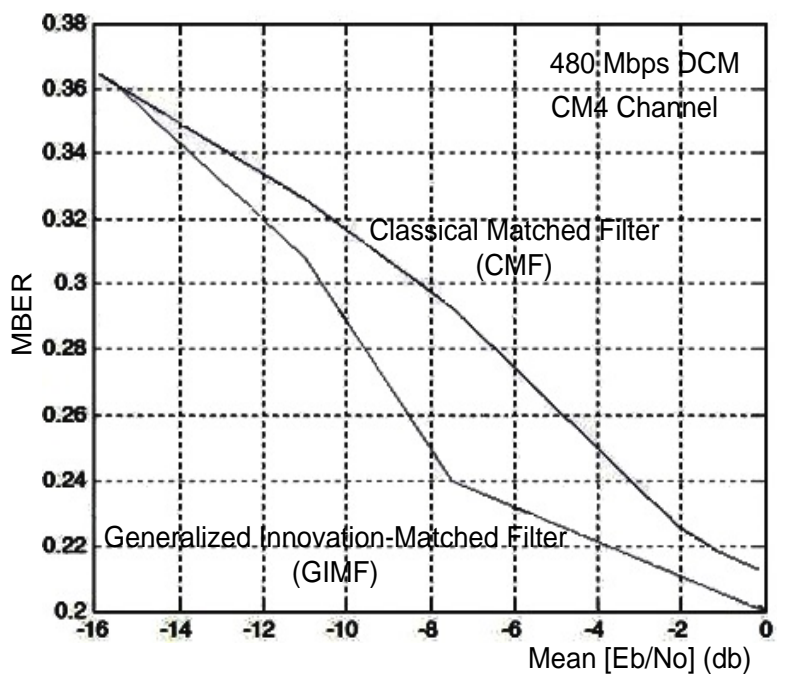

Figure 3. Estimated MBER of the CMF detector compared to GIMF for a Rayleigh fading channel with ACN and Gaussian PSD.

Figure 4 depicts the estimated MBER of the GIMF-processed 480 Mps-DCM signal for all UWB channels under ACN with a Gaussian-shaped power spectral density. The fading stochastic models (by order of severity) for CM4, CM3, CM2, and CM1 channels are shadow-lognormal, Nakagami- $m$, Rayleigh, and Rician, respectively. It is observed that CM1 enjoys the best performance due to the presence of a strong direct LOS, while CM4 has the largest MBER because it is governed by frequency selective fading and doubly stochastic shadow multipath. At low SNR margins, CM1 and CM2 channels exhibit a similar performance. It is also noted that the MBER values of CM3 and CM4 are very close. The analytical and experimental studies of MC-OFDM-UWB presented in this work can be extended to MIMO-OFDM [49-55].

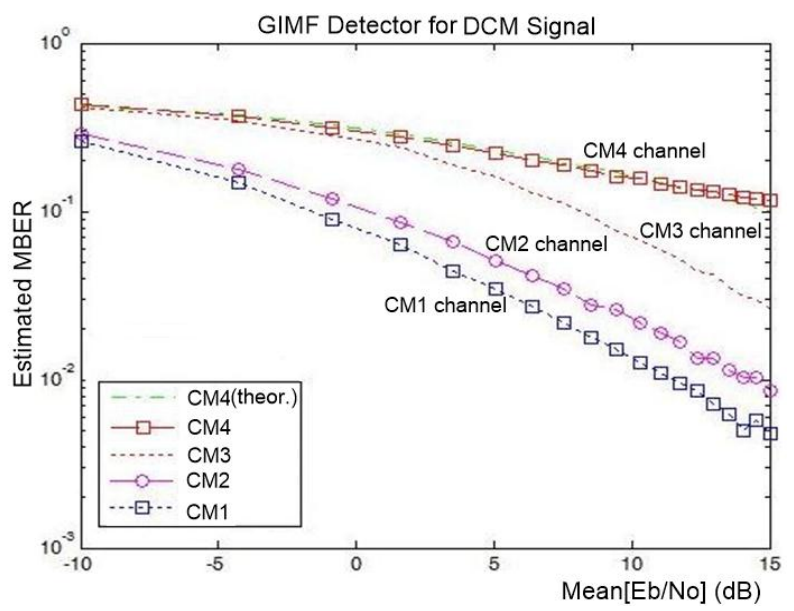

Figure 4. Estimated MBER for GIMF-detected 480 Mbps-DCM signal for Mode 1 CXs with ACN and Gaussian PSD.

\subsection{Comparative Analysis Between our Model and the CF-based Scheme at a RAKE Receiver}

The mean BER (MBER) versus average SNR per bit for different channel classes (CM1, CM2, CM3, CM4) is tabulated in Table 3. The MBER values of our reduced complexity Innovation-like receiver structure are compared to those obtained using the CF-based (characteristic function) computable model at a 10-finger RAKE receiver developed by Wang et al [56]. We observe that our model outperforms that of Wang et al with significant improvements occurring for CM1 and CM2 channels despite that fact the Wang's model employs a very large number of RAKE receiver fingers. For CM3 and CM4 channel, our model outperforms the CF-based structure for SNR below $8 \mathrm{~dB}$. As anticipated, CM1 channel has the best performance and is taken as a reference for comparison of SNR-gain. For 2\% and 3\% bit error rates, a $2 \mathrm{~dB}$-gain is achieved by our model.

Table 3. Comparison between the MBER of our model and the CF-based model at a 10-finger RAKE receiver

\begin{tabular}{|c|ccccccc|}
\hline SNR & $\begin{array}{c}1 \\
\mathrm{~dB}\end{array}$ & $\begin{array}{c}2 \\
\mathrm{~dB}\end{array}$ & $4 \mathrm{~dB}$ & $6 \mathrm{~dB}$ & $8 \mathrm{~dB}$ & $\begin{array}{c}10 \\
\mathrm{~dB}\end{array}$ & $\begin{array}{c}12 \\
\mathrm{~dB}\end{array}$ \\
\hline $\begin{array}{c}\text { Channel } \\
\text { Class }\end{array}$ & \multicolumn{6}{c}{ Mean Bit Error Rate (MBER) } \\
\hline $\begin{array}{c}\text { CM1 (our } \\
\text { model) }\end{array}$ & 0.07 & 0.06 & 0.04 & 0.03 & 0.02 & 0.013 & 0.009 \\
\hline $\begin{array}{c}\text { CM1 } \\
\text { (CF-RAKE) }\end{array}$ & 0.17 & 0.15 & 0.11 & 0.08 & 0.03 & 0.02 & 0.01 \\
\hline $\begin{array}{c}\text { CM2 (our } \\
\text { model) }\end{array}$ & 0.1 & 0.08 & 0.06 & 0.048 & 0.035 & 0.025 & 0.014 \\
\hline $\begin{array}{c}\text { CM2 } \\
\text { (CF-RAKE) }\end{array}$ & 0.2 & 0.18 & 0.15 & 0.1 & 0.05 & 0.03 & 0.015 \\
\hline $\begin{array}{c}\text { CM3 (our } \\
\text { model) }\end{array}$ & 0.23 & 0.2 & 0.176 & 0.137 & 0.11 & 0.08 & 0.05 \\
\hline $\begin{array}{c}\text { CM3 } \\
\text { (CF-RAKE) }\end{array}$ & 0.24 & 0.21 & 0.18 & 0.14 & 0.07 & 0.05 & 0.03 \\
\hline $\begin{array}{c}\text { CM4 (our } \\
\text { model) }\end{array}$ & 0.29 & 0.25 & 0.207 & 0.189 & 0.2 & 0.18 & 0.15 \\
\hline $\begin{array}{c}\text { CM4 } \\
\text { (CF-RAKE) }\end{array}$ & 0.3 & 0.26 & 0.21 & 0.19 & 0.12 & 0.09 & 0.06 \\
\hline
\end{tabular}




\section{Conclusions}

In this work, the performance of MB-OFDM in high-speed UWB was studied considering DCM modulation and UWB channels that conform to the IEEE 802.15.3a standard. A novel GIMF receiver was implemented as an extension to the classical RAKE structure. Analytical MBER expressions were derived and valided using simulated high-speed $480-\mathrm{Mbps}$ DCM signals over CM4, CM3, CM2, and CM1 UWB channels subject to shadow-lognormal, Nakagami-m, Rayleigh, and Rician fading statistics, respectively. All channels suffer from additive colour noise with Gaussian power spectral density. It was shown that the GIMF outperforms the classical matched filter due to its capability to adaptively capture the spectral characteristics of the interfering multi-user ACN and shape its power spectral density accordingly. Mean bit error rate results demonstrated the superior performance of CM1 channel, while the worst performance was attributed to the frequency selective and shadow driven CM4 channel. For low SNR margins, CM2 and CM1 channels exhibited a similar performance. For SNR margins below $0 \mathrm{~dB}$, the MBER values of CM4 were nearly identical to those of CM3.

A qualitative comparative analysis was also performed for the MBER that results at our Innovation-like receiver structure and at a 10-finger RAKE receiver [56] based on the CF computational model. It was found that our model outperforms that of the CF-based scheme for CM1 and CM2 channels for all SNR ranges, and for CM3 and CM4 channels for SNR values below $8 \mathrm{~dB}$. For MBER values of $2 \%$ and $3 \%$, our model achieves a $2 \mathrm{~dB}$-gain over the CF-based model even at a very large number of RAKE receiver fingers.

\section{Future Work}

UWB systems will be studied in channels governed by partially developed scattering $[57,58]$ driven by an underlying Poisson process with ARMA/AR rate [59-63]. In addition, machine learning detectors [64-67] will be incorporated into UWB receivers. Since this work assumed perfect channel state estimation, further extensions are planned to employ advanced channel estimation schemes [20], [30], [33-35] for the purpose of optimizing the standard deviation of the imperfect channel estimation. The implementation of UWB systems for position allocation [68] and early tsunami detection [69-72] will also be investigated. Finally, this work will be further expanded as a research book chapter to comprehensively cover UWB systems from a physical layer perspective [73].

\section{REFERENCES}

[1] J. S. Daba, "Performance of Ultra Wide Band Systems in High Speed Wireless Personal Area Networks," Advances in Electrical and Computer Technologies, LNEES, Springer Nature, pp. 634-650, 2019.

[2] T. W. Barrett, "History of Ultrawideband (UWB) Radar and Communications: Pioneers and Innovators," Proc. Progress in Electromagnetics Symposium (PIERS), Cambridge, MA, 2000.

[3] T. Barrett, "History of Ultra wideband Communications and Radar: UWB Communications," Microwave, 2001.

[4] J. Balakrishnan, A. Batra and A. Dabak, "A Multi-Band OFDM System for UWB Communication," IEEE Conference on Ultra Wideband Systems and Technologies, pp. 354-358, Nov. 2003.

[5] R. S. Kshetrimayum, "An Introduction to UWB Communic ation Systems," IEEE Potentials, 28(2), 2009.

[6] F. Nekoogar, Ultra-Wideband Communications: Fundamen tals and Applications, Prentice Hall, 2005.

[7] W. P. Siriwongpairat \& K. J. Ray Liu, $U W B$ Communications Systems: Multiband OFDM Approach, Wiley, 2007.

[8] M. Ghavami, L. Michael and R. Kohno, UWB Signals and Systems in Communication Engineering, Wiley, 2007.

[9] R. Tesi, M. Codreanu, I. Oppermann, "Interference Effects of UWB Transmission in OFDM Communication Systems," International Symposium on Ultra Wideband Systems, Oulu, June 2003.

[10] O. Abdul-Latif and J. P. Dubois, "Performance of UWB System in a Partially Developed Fading Channel with CCI," Proc. of the $5^{\text {th }}$ IEEE GCC Communication and Signal Processing Conf., Kuwait, pp. 1-5, 2009.

[11] J. P. Dubois, "Recent Advances in Wireless Systems," Annual Review of Wireless Communications: Volume 3, International Engineering Consortium (IEC), Chicago, 15 March, 2008.

[12] J. P. Dubois, "Recent Advances in Wireless MIMO Systems with Improved Receiver Diversity Combiners," in Annual Review of Communications: Volume 60, International Engineering Consortium (IEC), Chicago, pp. 513 - 527, 2007.

[13] J. P. Dubois and O. Abdul-Latif, "Novel Diversity Combining in OFDM-Based MIMO Systems," Proceedings of the American Conference on Applied Mathematics, Harvard University, pp. 189-194, 2008.

[14] J. Dubois and O. Abdul-latif, "Improved Receiver Diversity Processing over SIMO Fading Channels," IEEE International Conference on Signal Processing and Communications (ICSP'07), Dubai, Nov. 2007.

[15] J. Daba and M. R. Bell, "Object Discrimination and Orientation-Determination in Speckled Images," Optical Engineering, vol. 33, no. 4, pp. 1287-1302, April 1994.

[16] J. Daba and M. R. Bell, "Statistics of the Scattering Cross Section of a Small Number of Random Scatterers," IEEE Transactions on Antennas and Propagation, vol. 43, no. 8, 
pp. 773-783, August 1995.

[17] A. Abdi, S. Nader-Esfahani, J. Daba and M. R. Bell, "Comments on Statistics of the Scattering Cross Section of a Small Number of Random Scatterers," IEEE Trans. Antennas \& Propagation, vol. 48, no. 5, pp. 844-845, May 2000 .

[18] J. Daba and M. R. Bell, "Synthetic-Aperture-Radar Surface Reflectivity Estimation Using a Marked Point-Process Speckle Model," Optical Engineering, vol. 42, no. 1, pp. 211-227, January 2003.

[19] J. P. Dubois and O. Abdul-Latif, "Detection of Ultrasonic Images in the Presence of a Random Number of Scatterers: A Statistical Learning Approach," Int. Journal of Computer and Information Engineering, vol. 1, no. 12, 2007.

[20] J. P. Dubois and O. Abdul-Latif, "SVM-Based Detection of SAR Images in Partially Developed Speckle Noise," International Journal of Computer and Information Engineering, vol. 1, no. 12, 2007.

[21] J. P. Dubois, "Improved Segmentation of Speckled Images Using an Arithmetic-to-Geometric Mean Ratio Kernel", International Journal of Electrical and Computer Engineering, vol. 1, no. 10, 2007.

[22] J. Daba and M. R. Bell, "Segmentation of Speckled Images Using a Likelihood Random Field Model," Optical Engineering, vol. 47, no. 1, pp. 017005-1 to 017005-20, Jan. 2008.

[23] J. S. Daba and P. Jreije, "Advanced Stochastic Models for Partially Developed Speckle," International Journal of Electronics and Communication Engineering, vol. 2, no. 5, pp. 1001-1005, 2008.

[24] J. P. Dubois, "Statistical Modeling of Local Area Fading Channels Based on Triply Stochastic Filtered Marked Poisson Point Processes," Int. J. of Electronics and Communication Engineering, vol.9, no.7, 2015.

[25] J. P. Dubois, "Statistical Modeling of Mobile Fading Channels Based on Triply Stochastic Filtered Marked Poisson Point Processes," Int. J. of Electronics and Communication Engineering, vol. 11, no. 1, 2017.

[26] J. Daba and M. R. Bell, "Object Discrimination and Orientation-Determination in Synthetic Aperture Radar Images," IEEE Int. Geoscience and Remote Sensing Symp., Houston, vol. 2, pp. 877-880, May 23-29, 1992.

[27] J. Daba and M. R. Bell, "Statistical Distributions of Partially Developed Speckle Based on a Small Number of Constant Scatterers with Random Phase," Proceedings of the IEEE International Geoscience and Remote Sensing Symposium, California Institute of Technology, vol. 4, pp. 2338 - 2341, August, 1994.

[28] J. Daba and M. R. Bell, "Estimation of the Surface Reflectivity of SAR Images Based on a Marked Poisson Point Process Model," Proceedings of the IEEE International Symposium on Signals, Systems, and Electronics, San Francisco, CA, pp. 183-186, October 25, 1995.

[29] J. P. Dubois, "Segmentation of Speckled Ultrasound Images Based on a Statistical Model," EURASIP Proceedings of the 16th Biosignal Conference (Biosignal 2002), Brno, vol. 16, pp. 377-380, June 2002.

[30] J. P. Dubois, "Scattering Statistics of Doppler Faded Acoustic Signals Using Speckle Noise Models," IEEE International Conference on Direct and Inverse Problems of Electromagnetic and Acoustic Wave Theory (DIPED 2003), Lviv, Ukraine, pp. 185-189, Sept. 2003.

[31] J. P. Dubois, "Traffic Estimation in Wireless Networks Using Filtered Doubly Stochastic Point Processes", IEEE International Conference on Electrical, Electronic, and Computer Engineering, Cairo, Sept. 2004.

[32] J. P. Dubois, "Estimation Algorithms for Quantitative Tissue Characterization in Ultrasound Images Using Doubly Stochastic Translated Point Processes", IEE Proceedings of the 2nd International Conference on Advances on Medical Signal and Information Processing (IEE 2004), Valleta, pp. 274-280, Sept. 2004.

[33] J. P. Dubois, "Estimation of the SNR for Wireless Systems in a Local Fading Environment with Multi-Element Antennas," EURASIP Proc. of the $13^{\text {th }}$ Int. Conference on Signal Processing, Istanbul, Sept. 2005.

[34] J. P. Dubois, "Poisson Modulated Stochastic Model for Partially Developed Multi-Look Speckle," Proceedings of the American Conference on Applied Mathematics, Harvard University, 2008.

[35] J. Goodman, Speckle Phenomena in Optics: Theory and Applications, Roberts and Company Pub., 2007.

[36] J. P. Dubois and P. Jreije, "Probability Distributions for Multiplicative Noise in Internet Associated Wireless Cells and in Speckled Images," Proceedings of the Conference on Applied Mathematical Problems, Ukrainian Mathematical Congress, Kiev, Ukraine, pp. 246-253, August, 2009.

[37] L. Zhang and C. Yang, "The Equalization Performance of RAKE Receiver in UWB System," Proceedings of the $7^{\text {th }}$ International Conference on Signal Processing, Beijing, China, Sept. 2004.

[38] R. F. Pawula, S. O. Rice, and J. H. Roberts, "Distribution of the Phase Angle Between Two Vectors Perturbed by Gaussian Noise," IEEE Tran.s on Com., vol. COM-30, no. 8, pp. 1828-1841, August 1982.

[39] M. K. Simon and M. Alouini, Digital Communication over Fading Channels, Wiley-Interscience, 2005.

[40] M. K. Simon, S. M. Hinedi, and W. C. Lindsey, Digital Communication Techniques - Signal Design and Detection, PTR Prentice Hall, Englewood Cliffs, NJ, 1995.

[41] J. Lu, K. Letaief, J. Chuang, and M. Liou, "M-PSK and $M$-QAM BER Computation Using Signal-Space Concepts," IEEE Transactions on Communication, vol. 47, no. 2, pp. 181-184, Feb. 1999.

[42] R. Ayoubi and J. Daba, "FPGA Design of Spatially Modulated Single-Input-Multiple-Output Signals in $5 \mathrm{G}$ Diversity Receivers," Proceedings of the 8th IEEE International Conference on Communication, Networks and Satellite (COMNETSAT 2019), Makassar, Indonesia, August 2019.

[43] R. A. Ayoubi, J. S. Daba, and S. Berjawi, "FPGA Realization of MRC with Optimized Exponent for Adaptive Array Antennas," Proceedings of the 21st IEEE 
International Conference on High Performance Computing and Communications, Zhangjiajie, China, August 2019.

[44] S. K. Kondoju, V. V. Mani, and R. Bose, "Exact BER Analysis of DCM for Multiband OFDM-UWB System over Uncorrelated Nakagami-m Fading Channels," 2014 IEEE International Conference on Ultra-Wideband (ICUWB), pp. 473-478, Paris, France, Sept. 2014.

[45] N. Nouri, A. Messouidi, and R. Bouallege, "Performance Comparison DCM versus QPSK for High Data Rates in the MBOFDM UWB System," $8^{\text {th }}$ Int. Conf. on Networks and Communications, Spain, Dec. 2016.

[46] J. P. Dubois, R. Minkara, and R. Ayoubi, "Generalized Maximal Ratio Combining as a Supra-optimal Receiver Diversity Scheme", Int. J. of Electronics and Communication Engineering, vol. 4, no. 8, 2010.

[47] R. Ayoubi, J. P. Dubois, and R. Minkara, "FPGA Implementation of Generalized Maximal Ratio Combining Receiver Diversity", Int. J. of Electronics and Communication Engineering, vol. 4, no. 8, 2010.

[48] R. Minkara and J. P. Dubois, "Improved Root-Mean-Square-Gain-Combining for SIMO Channels," International Journal of Electronics and Communication Engineering, vol. 2, no. 5, pp. 798-801, 2008.

[49] J. S. Daba, J. P. Dubois and Y. Antar, "Peak Data Rate Enhancement Using Switched Micro-Macro Diversity in Cellular Multiple-Input-Multiple-Output Systems," International Journal of Electronics and Communication Engineering, vol. 11, no. 1, pp. 99-106, 2017.

[50] J. S. Daba, J. P. Dubois and G. El Soury, "Performance Analysis in $5^{\text {th }}$ Generation Massive Multiple-Input-Multipl e-Output Systems," $20^{\text {th }}$ International Conference on Ubiquitous Communication Systems, Paris, France, June, 2018.

[51] J. P. Dubois, and P. Jreije, “A Novel Receiver Diversity Combining Technique for Internet-Based 4G Wireless Communication", Proceedings of the International Conference on Recent Advances in Signal Processing, Robotics and Automation, University of Cambridge, United Kingdom, pp. 127-132, Feb., 2010.

[52] J. P. Dubois, "A New QoS Provisioning Technology and its Impacts on Future 4G Networks," in Annual Review of Communications: Volume 61, International Engineering Consortium (IEC), Chicago, pp. 601 - 615, 15 August, 2009.

[53] R. Ayoubi, J. P. Dubois, and O. Abdul-latif, "FPGA Implementation of a Novel Receiver Diversity Combining Technique for Wireless SIMO Systems," Proceedings of the IEEE International Conference on Signal Processing and Communications, Dubai, pp. 37-40, Nov. 2007.

[54] O. Abdul-Latif and J. P. Dubois, "LS-SVM Detector for RMSGC Diversity in SIMO Channels," Proceedings of the IEEE International Conf. on Information Sciences, Signal Processing and its Applications, Dubai, pp. 1-4, Feb. 2007.

[55] J. S. Daba, J. P. Dubois and O. Abdul-Latif, "Uplink Performance of Diversity Combining in MIMO Systems Using SVM Classifiers," ResearchGate Technical Report, pp. 1-8, November, 2018.

[56] L. Wang and W. Liu, "Bit Error Rate Analysis in IEEE 802.15.3a UWB Channels," IEEE Transactions on Wireless Communications, vol. 9, no. 5, pp. 1537-1542, May 2010.

[57] J. S. Daba and M. R. Bell, "Statistics of the Scattering Cross Section of a Collection of Constant Amplitude Scatterers with Random Phase," Purdue University Technical Report, pp. 1-37, 01 July 1994.

[58] J. S. Daba, Detection and Estimation in Speckled Images Based on Marked Point Process Speckle Noise Models, Ph.D Dissertation, Purdue University, West Lafayette, IN, USA, pp. 1-182, August 1994.

[59] J. P. Dubois, "Burstiness Reduction of a Doubly Stochastic AR-Modeled Uniform Activity VBR Video," International Journal of Electrical and Computer Engineering, vol. 1, no. 10, pp.1478-1482, 2007.

[60] J. P. Dubois and H. M. Chiu, "Statistical Multiplexing of VBR Video in ATM Using a Hybrid Markov Modulated Poisson Process and Fluid Approximation Model," Proceedings of the IEC'05, Prague, 2005.

[61] J. P. Dubois, "Modeling of Statistically Multiplexed Non Uniform Activity VBR Video," International Journal of Electrical and Computer Engineering, vol. 1, no. 10, pp. 1483-1489, 2007.

[62] J. P. Dubois and H. M. Chiu, "High Speed Video Transmission for Telemedicine Using ATM Technology," Int. Journal of Electrical and Computer Engineering, vol. 1, no. 12 , pp. 515-519, 2007.

[63] J. P. Dubois, "Congestion Control Mechanism of ATM Traffic Using Leaky Bucket VP Shaping," Proceedings of the IEEE International Conference on Trends in Communications (EUROCON'2001), Bratislava, vol. 2, July 2001.

[64] J. P. Dubois and O. Abdul-Latif, "Improved M-ary Signal Detection Using Support Vector Machine Classifiers," Proceedings of the IEC'05, Prague, Czech Republic, pp. 264-268, August 26-28, 2005.

[65] J. P. Dubois and O. Abdul-Latif, "Least Square-SVM Detector for Wireless BPSK in Multi-Environmental Noise," Int. Journal of Electronics and Communication Engineering, vol. 2, no. 8, pp. 1692-1697, 2008.

[66] J. P. Dubois and O. M. Abdul-Latif, "A Novel SVM-Based OOK Detector in Low SNR Infrared Channels", Int. Journal of Computer and Information Engineering, vol. 1, no. 7, pp. 1091-1095, 2007.

[67] J. S. Daba, O. M. Abdul-Latif, "Supervised Machine Learning Classifiers for Diversity Combined Signals in 6G Massive-MIMO Receivers," Universal Journal of Electrical and Electronic Engineering, Horizon Research Publishing, 2020.

[68] J. P. Dubois, M. Nader, and C. El Ferkh, "GSM Position Tracking Using A Kalman Filter," International Journal of Electronics and Communication Engineering, vol. 6, no. 8, pp. 867-876, 2012.

[69] J. P. Dubois, H. Karam, and J. Abdallah, "An Enhanced SAR-Based Tsunami Detection System," International Journal of Electronics and Communication Engineering, vol. 8, no. 7, pp. 1242-1246, 2014. 
[70] C. Intanagonwiwat, R. Govindan, and D. Estrin, "Directed SDiffusion: a Scalable and Robust Communication Paradigm for Sensor Networks," Mobile Computing and Networking, pp. 56-67, 2000.

[71] J. S. Heidemann, F. Silva, C. Intanagonwiwat, R. Govindan, D. Estri, and D. Ganesan, "Building Efficient Wireless Sensor Networks with Low-Level Naming," Symp. Operating Sys. Princ., pp. 146-159, 2001.
[72] C. Meinig, S. Stalin, A. Nakamura, F. Gonzelez, and H. Milburn, "Technology Developments in Real-Time Tsunami Measuring, Monitoring and Forecasting," Oceans 2005 MTS/IEEE, Washington, 2005.

[73] J. Daba, "Performance of Ultra Wide Band Systems in High Speed Wireless Personal Area Networks," Advances in Electrical and Computer Technologies, Lecture Notes in Electrical Engineering Series, Springer Nature, vol. 672, pp. 911-935, September 2020. 